\title{
La pesca de esturión en el reino de Valencia, Aragón y Cataluña (siglos XIV-XV)
}

\author{
The fishing of sturgeon in the Kingdom of Valencia, Aragon and \\ Catalonia (XIVth - XVth centuries)
}

\author{
Pablo José Alcover Cateura \\ A Gemma Avenoza Vera (1959-2021). \\ Maestra y directora de tesis.
}

\section{Author:}

Pablo José Alcover Cateura

Observatorio de la Alimentación (ODELA), Campus de la Alimentación de Torribera (Universitat de Barcelona).

palcovca@gmail.com

https://orcid.org/0000-0002-1558-3005

Date of reception: 16/04/21

Date of acceptance: 19/05/21

\section{Citation:}

Alcover Cateura, P. J. (2021). La pesca de esturión en el reino de Valencia, Aragón y Cataluña (siglos XIV-XV). Anales de la Universidad de Alicante. Historia Medieval, (22), 319-339.

https://doi.org/10.14198/medieval.19382

Acknowledgments: Agradezco los comentarios y sugerencias para este trabajo de mi director de tesis y maestro, Antonio Riera Melis (UB-IEC) y a Carlos Alfaro Zaforteza (King's College London).

C 2021 Pablo José Alcover Cateura

Licence: This work is licensed under a Creative Commons Attribution 4.0 International License (CC BY 4.0).

\section{RESUMEN}

Comer esturión era un claro indicador de rango social elevado. Su ingesta era utilizada para diferenciar las mesas de las clases altas de las no privilegiadas. Por ello, su pesca suscitó el interés de las élites, desde reyes a jurados y consejeros de ciudades y villas, quienes controlaron su captura. Los estudios de este pescado se han centrado en analizar su papel dentro de los sistemas alimentarios, su precio en las pescaderías y los restos óseos hallados en yacimientos arqueológicos. Sin embargo, no hay estudios que analicen su pesca, debido sobre todo a la escasez de fuentes escritas. El presente trabajo pretende realizar una primera aproximación a su captura dentro de la historiografía de la Corona de Aragón. El presente artículo selecciona las principales aportaciones en torno al esturión en el medievo y aporta nuevas fuentes cuya interpretación permite obtener las características generales de la pesca del selecto pescado. Además, se realiza una aportación desde la interdisciplinariedad, combinando resultados de la investigación en archivos y los más relevantes estudios de arqueofauna ibérica. Finalmente, se pretende llevar a cabo un estudio comparativo entre la captura de esturión en aguas fluviales de la 
Corona de Aragón con la del Reino de Inglaterra, de Hungría y Croacia y los Comuni para constatar elementos comunes.

PALABRAS CLAVE: esturión; Historia de la alimentación; pesca; libros del mostassaf.

\section{ABSTRACT}

Eating sturgeon was a powerful symbol of high social rank and a clear way to distinguish a privileged person from a no privileged. For this main reason, its fishing was controlled by kings and town council officers, by extension, the social elite. Studies about this fish have focus its attention in its role, played in the different food systems, its price in the fish markets and the archaeological remains. Nevertheless, there are not publications about its fishing, mainly to the fact that the scarcity of primary written sources. This paper wants to be the first approach to this specific issue, nowadays without researches in the Late Medieval historiography of the Crown of Aragon. The present paper picks on the main contributions on the capture of the sturgeon in Medieval times and adds new sources whose analysis enables a better overall understanding of the capture procedures of this select fish. This is an interdisciplinary contribution, which combines both archival research and the relevant studies on Iberian archeofauna. Finally, this essay attempts to make a comparative study of sturgeon capture procedures among the rivers of the Crown of Aragon, the Kingdom of England, Hungary, Croatia and the Comuni, to find differences and similarities.

KEY WORDS: sturgeon; Food History; fishing; mostassaf's books.

\section{INTRODUCCIÓN. FUENTES Y METODOLOGÍA}

El esturión era más que pescado que saciaba, aportaba un toque de distinción en las mesas $^{1}$. Su presencia en un banquete era una muestra de ostentación y riqueza por parte del anfitrión. Además, era la pieza más cara en una pescadería ${ }^{2}$. Sus consumidores más habituales eran los estamentos urbanos más económicamente solventes ${ }^{3}$.

1 Agradezco los comentarios y sugerencias para el presente artículo de Antonio Riera Melis (UB-IEC), mi maestro y antiguo director de tesis, y de Carlos Alfaro Zaforteza (King's College London). Además, quiero agradecer los comentarios de los dos revisores anónimos, que ayudaron a mejorar este trabajo.

2 La historiografía que realiza análisis comparativos de precios de distintos pescados, emitidos mediante ordenanzas por los gobiernos locales en Cuaresma, es extensa (Cave, Coulson, 1965, p. 405; Santamaría, 1966, p. 54; Lanconelli, 1985, pp. 83-131; Mutgé Vives, 2004, p. 257; Lanconelli, 2005, pp. 181-204; Pucci Donati, 2017, pp. 40-43; Lleonart, Maynou, Salicrú i Lluch, 2018, p. 164; Bartosiewicz, Biller, Csippán, Daróczi-Szabó, Daróczi-Szabó, Gál, Kováts, Lyublyanovics, ÁgnesNyerges, 2018, p. 140; Macheridis, Hansson, Foley, 2020, pp. 1024-1080).

3 Es abundante la bibliografía sobre el papel de los pescados, incluido el esturión, dentro de los distintos sistemas alimentarios (Montanari, 1985, pp. 634-637; Montanari, 1988, pp. 43-45, 81; Montanari, 1989, pp. 74, 254, 307; Flandrin, 1992, pp. 173-1992; Montanari, 1993, pp. 103, 224; Flandrin, 1993, pp. 177- 
La pesca de esturión es un aspecto muy poco estudiado ${ }^{4}$. Se han realizado análisis de restos óseos, las evidencias arqueofaunísticas. En general, estos estudios se centran en aportar datos que ayudan a comprender mejor la evolución histórica del pez, con el objetivo de contribuir a su preservación actual, hoy casi extinto ${ }^{5}$.

El presente artículo desea ser la primera aportación en la historiografía medieval española cuyo objeto de estudio principal es realizar una aproximación a la pesca de esturión. También se pretende relacionar su captura con la realizada para esta misma especie en otros estados, especialmente, el Reino de Inglaterra, de Hungría y Croacia y los Comuni. Los aspectos básicos que se propone resolver son qué especie de esturión se capturaba, las zonas y artes de pesca y si había o no libertad de pesca y porqué.

Las fuentes escritas más usadas son recopilaciones de ordenanzas. Se ha analizado el contenido de 107 manuscritos. De estos, 97 forman un corpus homogéneo al ser todos libros del mostassaf (29 editados y 68 inéditos), bases documentales de mi tesis doctoral (Alcover Cateura, 2019, pp. 405-428) ${ }^{6}$. Las obras restantes son tam-

192; Riera Melis,1994, p. 198; Trenchs Odena, 1995, pp. 209-328; Curtó Homedes, 1995, pp. 149-166; Riera Melis, 1996, p. 192; Hernández Íñigo, 1997, p. 1053; Giagnacovo, 2002, p. 194; Querrien, 2003, pp. 409-435; de Castro Martínez, 2004, p. 319; Alanyà Roig, 2005: 429-484; Serrano Larráyoz, 2005, pp. 205-206, 323; Flandrin, 2007, pp. 34-42; Rodrigo Estevan, 2009, p. 550; Riera Melis, 2009, pp. 133-135; Guerrero Navarrete, 2009, pp. 243, 260; di Schino, 2010, pp. 538-571; Riera Melis, 2013, pp. 65-98; Riera Melis, 2014, pp. 23-80; Riera Melis, 2016, pp. 21-52; Riera Melis, 2017, pp. 21-66; Riera Melis, 2018, pp. 17-84; Giagnacovo, 2018, pp. 229-264; Stunault, 2019). En la historiografía del reino de Valencia destacan los estudios sobre alimentación de los privilegiados de Juan Vicente García Marsilla, pero en ellos no se analiza el esturión al no hallarse ninguna referencia en las fuentes estudiadas por el autor (García Marsilla, 1993; García Marsilla, 2010; García Marsilla, 2018, pp. 1-26).

4 La bibliografía sobre la pesca en la baja Edad Media es vasta (Thomazi, 1947, pp. 284-287; Stouff, 1970, p. 203; Grava, 1980, pp. 48-58; Amargier, 1980, pp. 68-83; Malpica, 1984, pp. 103-117; Hinojosa Montalvo, 1987, pp. 159-173; Mollat du Jourdin, 1987; de Oliveira Marques, 1987, pp. 111; Aston, 1988, pp. 74-77; Ferreira Priege, 1988; Montanari, 1993, pp. 103, 224; Teña García, 1995, pp. 111-134; Hinojosa Montalvo, 1995a, pp. 191-204; Hernández Íñigo, 1997, p. 1053; Felisati, 1998, pp. 358, 428; Arizaga Bolumburu, 2000, pp. 13-28; Kowaleski, 2000, pp. 429-454; Aznar Vallejo, 2000, pp. 229-240; Querrien, 2003, pp. 409-435; Abad García, Peribáñez Otero, 2006, pp. 147-180; Sánchez Quiñones, 2006, pp. 145-169; Blair, 2007, pp. 47-49; Gresser, 2008; Riera Melis, 2009, pp. 133-135; Ferreira Priege, 2009, pp. 11-34; Bello León, 2009, pp. 35-54; Álvarez Fernández, 2009, pp. 71-86; Malpica, 2009, pp. 193-220; Medrano Fernández, 2009, pp. 221-234; Igual Luis, 2010, pp. 68-84; Kowaleski, 2010, pp. 23-28; Azevedo Pereira, 2012; Delsalle, 2013, pp. 95-118; Kowaleski, 2015, pp. 43-59; Hoffmann, 2016; Bartosiewicz et al, 2018, pp. 141-142; Montanari, 2019, pp. 84-85; Igual, 2020, pp. 49-70; Sweetinburgh, 2020, pp. 80-99; Tavares, 2021, pp. 44-57).

5 Entre los estudios arqueofaunísticos del esturión en la Península Ibérica y historiografía especializada europea se destaca: Morales-Muñiz, Rosselló-Izquierdo, Lentacker, Morales-Muñiz, 1994, pp. 453-475; Rosselló-Izquierdo, Morales-Muñiz, Morales-Muñiz, 1994, pp. 323-331; Locker, 2000, p. 78; Jones, Laughton, Clark, 2000, pp. 106-113; Barrett, Locker, Roberts, 2004, pp. 2417-2421; Davis, 2006; Bartosiewicz, Bonsall, 2008, pp. 35-45; Morales-Muñiz, Rosselló-Izquierdo, Morales-Muñiz, 2009, pp. 153-154, 160-62; Williot, Patrick, Rouault, Thierry, Brun, Rémi, Gressner, Jörn, 2011, pp. 165-174; Ludwig, Morales-Muñiz, Rosselló-Izquierdo, 2011, pp. 131-141.

6 Todos contienen normativa datada entre los siglos XIV y XV. 
bién volúmenes que contienen normas locales ${ }^{7}$. La información obtenida a partir de su estudio se complementa con los resultados de las investigaciones de arqueofauna. Por último, se incluye documentación editada e inédita relativa al derecho de regalía de los reyes sobre los pescados reales.

El presente artículo no incluye estudios detallados sobre la fiscalidad presente en el circuito urbano que centralizaba la venta de esturión, ni de fuentes sobre aranceles de aduanas, registros de generalidades, lezdas y peajes ${ }^{8}$. Tampoco se analizan memoriales de cuentas de los miembros de la Casa de Barcelona, de la alta jerarquía eclesiástica y nobiliaria y manuscritos de alta cocina, como recetarios y artes cisorias, así como obras de temática médica y alimentaria, particularmente regímenes de sanidad $^{9}$. Además, no se han consultado obras de Llull, Eiximenis, Ferrer, Roig, Turmeda y Marc, entre otros autores literarios, que aportan información relativa a la pesca y el consumo de pescado, puntualizando la otra documentación (Aparici Romero, 2012, pp. 13-24). Estas fuentes serán analizadas en dos futuras publicaciones, la primera sobre venta y la segunda relativa al papel del esturión en los sistemas alimentarios de la Corona de Aragón (siglos XIV-XV). Así, este trabajo son los primeros resultados de una investigación en curso, centrada en realizar una aproximación sobre la captura de este pescado selecto. Esta línea de investigación se inició durante mis cinco años de investigación doctoral y, se decidió, siguiendo las recomendaciones de mis directores de tesis, que fuera material de trabajo posterior a la defensa. Por último, en este artículo se utiliza indistintamente esturión o sollo porque son sinónimos en los siglos XIV y XV en la Península Ibérica, como apunta Miguel Gual Camarena ${ }^{10}$.

\section{LA PESCA}

El esturión no era el pescado generador de mayor riqueza en la Corona de Aragón. Sardina, anchoa, merluza y atún eran las especies más demandadas (Salicrú Lluch, 1995, pp. 29-85). Sin embargo, el sollo tuvo un papel relevante, más cultural que económico, al ser considerado el pescado más selecto. No hubo una industria de-

7 Todos son obras editadas con normativa datada entre los siglos XIV y XV (Revest Corzo, 1957; Guix Sugranyés, 1963; Icart, 1982; Massip Fonollosa, 1996; Guinot Rodríguez, 2006; Muntané i Santiveri, 2014).

8 Se destacan las aportaciones de José Sesma Muñoz, María Elisa Ferreira Priege, David Igual Luis y Roser Salicrú Lluch sobre estas fuentes sobre comercio de larga distancia (Sesma Muñoz, 2005; Sesma Muñoz, 2006; Sesma Muñoz, 2010; Ferreira Priege, 1984; Ferreira Priege, 1988; Igual Luis, 2007, pp. 377-309; Salicrú Lluch, 1995).

9 Antoni Riera Melis analiza algunos memoriales de cuentas, llibres de messió de casa y constituciones cibariorum y estudia la normativa de las Leges palatinae y Ordinacions de la Casa y Cort. Explica la información que aportan y que no aportan y en qué contexto concreto deben comprenderse estas fuentes (Riera Melis, 2015, pp. 22-24; Riera Melis, 2017, pp. 23-25; Riera Melis, 2018, pp. 31-38).

10 Gual de Camarena concluye que el término sollo en las fuentes jurídicas castellanas (siglos XII-XV) y el es, sin lugar a dudas, el esturión (Gual de Camarena (2013), s. v. "sollo"). 
dicada a su captura y exportación, como sí sucedía, por ejemplo, con la sardina y merluza. No obstante, sí suscitó, como se intenta demostrar en este apartado, un interés por parte de la monarquía, los jurados y consejeros por controlar su pesca, debido, sobre todo, a que era un alimento que era un claro indicador de rango social elevado. En consecuencia, se comparte la hipótesis de David Igual Luis (Igual Luis, 2020, pp. 63-64) quién considera que la pesca no puede ser comprendida sólo desde la economía, sino que formaba parte de un todo relacionado. Por lo tanto, las preferencias alimentarias de las clases altas es un aspecto que se considera clave para el entendimiento de la captura del esturión.

Primero, es necesario resolver qué especie se capturaba. Los resultados obtenidos por los estudios arqueofaunísticos son unánimes: se trata de esturión común europeo (Acipenser sturio), el más extendido por Occidente. Este se integraba en el ecosistema de los ríos de la Corona de Aragón en la baja Edad Media. Venía estacionalmente a reproducirse a las aguas turbias y tranquilas de escaso calado, ricas en flora fluvial y situadas en el curso alto. Cada año, entre marzo y mayo, llegaba para desovar, aunque desde finales de enero llegaban los primeros ejemplares. Su temporada alta era entre mediados de marzo y la última semana de mayo, cuándo llegaban los ejemplares más grandes (Carreras Candi, 1993, p. 161; Williot et al., 2011, pp. 165-174; Ludwig, et al., pp. 131-141; Bartosiewicz et al, 2018, pp. 139-142) ${ }^{11}$.

Los cocineros profesionales conocían la estacionalidad del sollo. Ejemplo de ello es su inclusión en la lista de pescados del Llibre de totes maneres de potatges, uno de los principales recetarios de la cocina de los privilegiados, dónde se explica al lector la mejor época del año para comprar los más selectos alimentos de abstinencia. En ella, el esturión se recomienda adquirirlo entre abril y mayo ${ }^{12}$. Por tanto, el desconocido autor del Llibre recomendaba su compra cuando era más abundante y los ejemplares eran más grandes (Barrieras, Santanach, Riera, Fundació Alícia, 2017, p. 311).

El esturión se reproducía por huevas. Tras la eclosión, el alevín permanecía dos o tres años en el mismo sitio o en el estuario. Posteriormente, nadaba con dirección a alta mar, que habitaba hasta su época de reproducción, cuándo volvía al mismo lugar de cría. El período de madurez sexual del macho era entre los catorce y veintidós años y el de la hembra entre los dieciocho y cuarenta y ocho años. En ambos casos,

11 Este especie, también arribaba a otros ríos de la Península Ibérica. En el Calendario de Córdoba (siglo X) se explica la llegada de sollos al Guadalquivir y otros ríos del sur en primavera. También las crónicas andalusíes aportan datos de su pesca en el Ebro. Una crónica redactada bajo el califato de Abderramán III narra la captura de unos pescados que sobrepasan el quintar (41 kilos) y que arriban al Ebro desde el mar cada marzo. Joaquín Vallvé los identifica como esturiones (Lévi-Provençal, 1957, p. 171; Arié, 1982, pp. 237, 285; García Sánchez, 1983-1986, p. 259; Navarro, 1990, p. 268; Morales-Muñiz et al, 1994, pp. 453-475; Dufourcq, 1995, p. 118; Toledo Jordán, 1998, p. 172; Bramon, 2000, pp. 81-89; Vallvé, 2003 , p. 44; Bramon, 2017, pp. 53-54). Agradezco a Dolors Bramon algunas recomendaciones bibliográficas de la pesca de sollo en época andalusí.

12 El Llibre de totes maneres de potatges se ha datado entre finales del siglo XIV e inicios del siglo XV (Santanach, 2017, pp. 67-91). 
la primera vez que retornaban al río medirían aproximadamente dos metros (entre l'80 - 2'10 m) y pesarían unos $40 \mathrm{~kg}$. Los ejemplares de mayor edad que cada año venían a reproducirse podían superar los $100 \mathrm{~kg}$, y en casos excepcionales, los 300 $\mathrm{kg}$. A pesar de sus grandes dimensiones eran inofensivos. Los adultos se alimentaban básicamente de moluscos, crustáceos y peces pequeños (Granado Lorencio, 1995, p. 276; Velasco Marcos, 1997, p. 117; Vallvé, 2003, p. 44; Williot et al, 2011, pp. 165174; Ludwig et al, 2011, pp. 131-141; Ordeix, Solà, Bardina, Casamitjana, Munné, 2014, p. 32).

Su distribución geográfica principal era el Ebro, desde Tortosa a, como mínimo, Zaragoza. En los otros ríos es bastante más difícil documentar su captura. Sin embargo, las fuentes analizadas aportan indicios. Los libros de mostassaf y otras recopilaciones de ordenanzas incluyen listas de precios de pescados frescos, capturados en las aguas del término municipal y que debían venderse obligatoriamente en la pescadería durante la Cuaresma ${ }^{13}$. Así, la mención de esturión indicaría que era una captura local. A partir del estudio de esta documentación, las evidencias de arqueofauna y el análisis de las fuentes de derechos de regalía del rey sobre el esturión, es plausible plantear la existencia de pesca de sollo en el Segura, Turia, Júcar, Llobregat, Segre, Ter, Fluvià y Muga ${ }^{14}$.

13 Las zonas de pesca dentro del término municipal están reguladas a menudo por los libros de mostassaf. Por ejemplo, una ordenanza del año 1390 legisla que las zonas de pesca de Barcelona eran de Montgat a Castelldefels y doce lenguas desde la costa (Bajet Royo, 1994, p. 370).

14 La captura de esturión en el Ebro ha conservado bastantes referencias documentales, sobre todo en Tortosa, publicadas la mayoría por el archivero Enrique Bayerri y Bertomeu en los ochos volúmenes de su Historia de Tortosa y su comarca (Bayerri Bertomeu, 1935, pp. 150-154; Bayerri Bertomeu, 1960, p. 619). El resto de referencias permiten obtener indicios sobre otras zonas de pesca en ríos de la Corona de Aragón (ACA, Cancillería, registros, nº58, fol. 63v-65v; ACB, Pia Almoina, Sitges, Capbreus, 1409, f. 37v; AMGi, Fons Ajuntament de Girona, Llibre del mostassaf, RG 17490, f. 129r; Marco Baidal, 1960, p. 90; Santamaría, 1966, p. 54; Canellas, 1972, pp. 87-89; Icart, 1982, pp. 67-68; Lloris, Rucabado, Cerro, Demestre, Portas, Roig, 1984, p. 51; Carreras Candi, 1993, p. 161; Bajet Royo, 1994, p. 391; Curtó Homedes, 1995, pp. 149-166; Bramon, 2000, pp. 81-89; Mutgé Vives, 2004, p. 257; Alanyà Roig, 2005: 429-484; Furió, Garcia Oliver, 2007, p. 148; Sobrequés Callicó, 2008, p. 97; Rodrigo Estevan, 2009, p. 550; Riera Melis, 2009, pp. 133-135; Ordeix et al., 2014, p. 32; Ludwig et al., pp. 131-143; Williot et al., 2011, pp. 165-174; Mercader, Lloris, Rubacabo, 2001, pp. 24, 84; Colón Domènech, 2001, pp. 97-98; Cuellas Campodarbe, 2016, p. 176; Lleonart et al., 2018, p. 164; Alcover Cateura, 2022. Aunque es un autor tardío, Gaspar Juan Escolano (1560-1619), comenta en su Historia de Valencia que las aguas del reino valenciano son ricas en pesca, sobre todo las meridionales. Entre los peces que destaca el autor está el esturión, que, junto a otros anádromos, remonta los ríos de la gobernación de Orihuela (Escolano, 1980, p. 387). La fachada marítima de Orihuela tenía abundante pesca durante los siglos XIV y XV. Esto no atrajo la atención de los habitantes de la población por los oficios del mar. El porqué: la distancia que separaba la villa de las pesquerías (unos $27 \mathrm{~km}$ aproximadamente) y los peligros del mar. En Cuaresma, la demanda local de pescado aumentaba mucho, pero no había suficiente oferta en el mercado. Para solucionar el problema, entre otras medidas, los jurados de la localidad (1447) ofrecieron veinte florines a los que capturasen grandes peces, entre los que podría estar el sollo (Hinojosa Montalvo, 1987, pp. 169-170; Hinojosa Montalvo, Barrio Barrio, 1992, p. 564; Hinojosa Montalvo, 1995a, p. 196; Hinojosa Montalvo, 1995b, p. 104; Hinojosa Montalvo, 1995c, p. 507-525; Hinojosa Montalvo, 1996, pp. 209-222). 
El punto del curso de los ríos dónde era más abundante su pesca era la zona más próxima a un azud o presa, porque las manadas de sollos no podían seguir avanzando y se acumulaban en las aguas cercanas, dónde desovaban, al no tener otra opción. En los ríos de la Corona de Aragón había bastantes presas, azudes, pontones, empalizadas, acequias, canales y otras construcciones cuya función era el control humano del agua dulce. Toda esta arquitectura provocaba la interrupción del ciclo de reproducción de los esturiones, al dificultar o incluso impedir arribar a aguas de desove (Bayerri Bertomeu, 1935, pp. 150-154; Bayerri Bertomeu, 1960, p. 619; Marco Baidal, 1960, p. 90; Carreras Candi, 1993, p. 161; Williot et al., 2011, pp. 165-174; Ludwig et al., 2011, pp. 131-143).

Algunos ejemplos sobre presas y azudes construidos durante la baja Edad Media y que supusieron graves golpes para esta especie: vecina a la población de Cherta, en el bajo Ebro, se comenzó a construir una presa iniciada en el año 1443, en base a un azud anterior finalizado a comienzos del siglo XV. Se conocen las medidas de este último: 4 metros de alto y 310 metros de longitud (Querol Gómez, 2004, p. 41; Carreras Candi, 1993, p. 161; Ordeix et al., 2014, p. 32). En el caso de remontar su cauce por Tudela o llegar a través de uno de sus afluentes, se colocaban a menudo pontones y empalizadas que dejaban pasar la corriente, pero no los peces grandes. Además, el desvío de agua para regar, mediante acequias y canales, mermó aún más el fácil nado y remonte del río por parte de estos corpulentos seres marinos (Argudo Périz, 1997, p. 56).

Otro azud que afectó notablemente a la población de esturiones fue el del Júcar, cercano a las poblaciones de Cullera y Sueca. Se alzó en el año 1453 con la intención de usar las aguas para el riego de arrozales y otros cultivos, como cereales (Sanchis Ibor, 2011, pp. 88-90). Un último ejemplo es el Azud Mayor del Segura, que imposibilitó el paso de sollos hacia el curso alto. Los ejemplares entraban por Guardamar, pasaban por Orihuela y se detenían en el azud, situado junto a Javalí Nuevo, población del Reino de Murcia (de los Llanos Martínez Carrillo, 1997, pp. 17-20)15.

Tanto en las corrientes fluviales de la Corona de Aragón, antes citadas, como en el Rin, el Danubio y el estuario del Gironda, entre otros, cercanas a un azud o presa, los pescadores disponían una red muy fina y larga de orilla a orilla, que podía llegar a los 30 metros de largo, conocida como la brugina en Cataluña y los tirs plans en el Reino de Valencia ${ }^{16}$. En ambos estados, tenía plomadas en su base y flotadores de corcho en la parte superior. Así se mantenían erguida en el agua. Un grupo de pescadores, con la ayuda de sus familias, se acercaban a la red con pequeñas barcas y la

15 Es en este río donde se ha documentado la presencia más antigua de esturión en el Litoral valenciano y catalán. En el yacimiento fenicio de la Fonteta (Guardamar) se han hallado restos óseos de sollos (Ludwig et al., 2011, pp. 133).

16 En Piacenza (Italia) esta extensa red era conocida como la "maggiura" (Venditelli, 1992, p. 396) y era utilizada para capturar esturiones en el río Po. Se documenta desde el siglo XIII. 
iba tirando hacia sí, extrayendo sollos y otros peces enredados en ella. También se colocaba esta red en la desembocadura de un gran río, como es el caso de la Albufera de la ciudad de Valencia. Aunque una fuente tardía, en los Col.loquis de la Insigne Ciutat de Tortosa de Cristófor Puig (1510-c.1574), se describe la captura en el azud del Cherta con la "reyna de totes les exàrcies, la qual té mil y dos-centes brases de llargària", es decir, la brugina. Dicha red estaba particularmente pensada para capturar sollos y no dañarlos ni herirlos para que llegaran inmaculados al mercado local, a pesar de que también eran apresados otros pescados, como salmones, truchas, carpas y lisas (Duran, 1981, p. 187; Sanmartín Arce, 1982, p. 19; Venditelli, 1992, p. 396; Carreras Candi, 1993, p. 161; Ordeix et al., 2014, p. 32; Nienhuis, 2008, pp. 208-228; Ordeix et al., 2014, p. 32; Haidvogl, Lajus, Schmid, Pont, Jungwirth, Lajus, 2014, pp. 500-506; Bartosiewicz et al., 2018, pp. 139-142).

No todos los pescados selectos de la Corona de Aragón recibían este trato durante su captura. Los delfines, autóctonos de las costas levantinas en el bajo medioevo, eran mamíferos que adquirían elevados precios en pescaderías locales, como la de Alicante, Valencia, Castellón, Ciudad de Mallorca, Montblanc, Tortosa, Gerona y Cagliari, entre otras. Estos animales eran violentamente arponeados, y también a menudo apaleados y apedreados, por ser enemigos naturales de pescadores, al romper sus redes para comerse los peces ${ }^{17}$. El sollo, al no ser considerado un adversario para las gentes de mar y tener alto valor comercial era capturado con un arte distinta a la de otras especies valiosas y selectas, pescadas salvajemente y que no se preocupaban de la presentación de la pieza cuando llegada al mercado (Alcover Cateura, 2021).

El valor comercial de los pescados en los mercados municipales de la Corona de Aragón ha sido analizado por Antonio Riera Melis. Este autor ha explicado que existía una rígida jerarquía de pescados según el precio, dividida en seis niveles (Riera Melis, 2014, pp. 61-62). Estos se ordenarían, entre otros, debido a dos factores principales: el gusto y el tamaño de la pieza. En ella, el esturión se situaba en la cima y el pescado de roca en la base, al ser el más económico.

La documentación analizada coincide unánimemente en situar al esturión como el más caro, confirmando así la hipótesis de Riera Melis. En las distintas listas de precios de la ciudad de Valencia (1324), Castellón (1381, 1392, 1393), Tortosa (1342), Reus (c. 1470), Barcelona (1390, 1396, 1397, 1399, 1401, 1405, 1409, 1428, 1431, 1441, 1443, 1458, 1468, 1473, 1485), Balaguer (1313) y Gerona (1474), el sollo es la captura más costosa ${ }^{18}$. En suma, este pescado era un alimento más vinculado al

17 Estos precios se legislaban en ordenanzas emitidas por los concejos de las poblaciones mencionadas. Se datan entre los años 1369 y 1485.

18 Los manuscritos consultados, editados e inéditos, son respectivamente los siguientes: Santamaría, 1966, p. 54; Furió, Garcia Oliver, 2007, p. 148; Colón Domènech, 2001, pp. 97-98; Curtó Homedes, 1995, pp. 149-166; Guix Sugranyés, 1963, p. 59; Bajet Royo, 1994, p. 391; Mutgé Vives, 2004, p. 257; Cuellas Campodarbe, 2016, p. 176; AMGi, Fons Ajuntament de Girona, Llibre del mostassaf, RG 17490, f. 129r). 
mercado de lujo que al de productos básicos, de aquí el interés del pescador para que la pieza llegara entera y sin mácula a la pescadería local.

En otros estados, el sollo tenía también el precio más elevado del mercado local de pescado. En Roma, según los statuta pescivendulorum urbis (1405), el esturión del Tíber era el más caro de toda la oferta de pescados y mariscos (Lanconelli, 1985, pp. 83-131; Lanconelli, 2005, pp. 181-204). En Bolonia, según los statuta datados en los años 1335, 1352, 1357, 1376 y 1389, el sollo del Po era el más costoso en la Cuaresma (Pucci Donati, 2017, pp. 40-43). Por último, en Zsolca (Reino de Hungría y Croacia), el esturión del Sajó era el más valioso, según la lista de precios de pescado del año 1329 (Bartosiewicz et al. 2020, p. 140).

No había libertad de pesca del sollo porque era uno de los pescados reales. Sobre él la monarquía tenía unos derechos de regalía que podían transmitirse a personas e instituciones privadas. El rey Alfonso I (1073-1134) concedió en el Fuero de Zaragoza (c. 1124) libertad de pesca a los pobladores de la ciudad aragonesa, a excepción de la captura de esturiones, que debían ser entregados al merino real (Canellas, 1972, 87-89; García Edo, 2016, 173). En 1287, un privilegio real concedió el aprovechamiento de los esturiones pescados en el Ebro dentro del término municipal de Zaragoza, en ausencia del rey, quién se los reservaba para su mesa, a los frailes del convento de predicadores de la ciudad (Rodrigo Estevan, 2009, p. 550). En 1336, Pedro IV (1319-1387) confirmó el derecho de pesca de esturión en su ausencia al citado convento (ACA, Cancillería, registros, nº858, ff. 63v-65v).

En el traslado de la lezda de Colliure realizado en la corte de Sancho de Mallorca (1317) se ordena que la mitad de los esturiones capturados por los pescadores locales eran para el soberano, quién a cambio les obsequiaba con "I quortó" de buen vino y un pan común (Sobrequés Callicó, 2008, p. 97). Por otra parte, los administradores de la Almoina de los pobres de la Seo de Barcelona tenían el derecho de quedarse la mejor de cinco partes de cualquier sollo capturado en el término de la villa de Sitges desde 1326, cuándo se consiguió el señorío jurisdiccional, que originalmente era de propiedad real (ACB, Pia Almoina, Sitges, Capbreus, 1409, f. 37v; Batlle, Parés, 1986-1987, pp. 153-177; Baucells Reig, 1990, pp. 17-53).

Un último caso sobre la falta de libertad de pesca del sollo es en la Albufera de Valencia, el lugar por dónde entraban los esturiones desde el mar hacia el curso alto del río Turia. La Albufera era una de las fincas reales, cuya propiedad se documenta desde 1238. Uno de las vías de ingresos principales para el soberano era el cobro del derecho de regalía, el quint del pescado que, en principio, era un pago a través de la entrega de la quinta parte de las capturas, incluido el esturión. Este tributo se documenta pocos años después de la conquista de la ciudad de Valencia y estuvo vigente, como mínimo, hasta final del siglo XV (Caruana Tomás, 1954, pp. 230-240; Rosselló Verger, 1995, pp. 107, 141- 142; Freire Moliner, 1995, pp. 60-62, 162-163; Momblanch y Gonzálbez, 2003, pp. 235-250). 
Tampoco había libertad de pesca en otros estados europeos. A partir del reinado de Eduardo II de Inglaterra (1284-1327), el esturión fue considerado un pescado real. Según el privilegio De rogativa regis, cuándo se capturase un ejemplar debía entregarse por los pescadores a un oficial real (Johnson, 2019, pp. 100-103; Kowaleski, 2001, pp. 2-26). Segismundo II (1387-1437), rey de Hungría, Croacia y Bohemia, ordenó requisar dos enormes sollos que se habían capturado en el río Tisza, cerca de la ciudad de Eger. Los pescadores que los capturaron intentaron venderlos en el mercado local, pero fueron apresados por un oficial de soberano, quién consiguió que le fueran entregados después de enfrentarse a ellos (Bartosiewicz et al. 2020, p. 140). Por último, un privilegio del pontífice Gregorio IX datado en el año 1233, y vigente, como mínimo, hasta finales del siglo XV, otorgaba a la Abadía de Santa María de Grottaferrata el uso exclusivo de la única pesquería para capturar esturiones en el Tíber (Venditelli, 1992, p. 395).

\section{CONCLUSIONES}

El control de la pesca de esturión por parte de monarquías y poderes locales, tanto en la Corona de Aragón como en otros estados occidentales, buscaba particularmente asegurar el abastecimiento de un alimento que era un indicador de rango social elevado. Por una parte, la monarquía a través del derecho de regalía se reservaba una parte o la totalidad del ejemplar, impidiendo su libertad de pesca y asegurándose así su continuo abastecimiento. Por parte de jurados y consejeros, el control sobre las zonas de pesca, la actividad de pescadores y la centralización de las redes de distribución en la pescadería, permitía a las clases altas urbanas acceder a un pescado cuya ingesta era un símbolo de ostentación de su estatus social.

El río dónde se capturaban más sollos era, sin duda, el Ebro. También hay indicios documentales suficientes para considerar que se pescaban en otros ríos, como el Segura, Turia, Júcar, Llobregat, Segre, Ter, Fluvià y Muga. En consecuencia, desde las costas de la gobernación de Orihuela al condado de Ampurias era habitual la llegada estacional de estos peces colosales.

La zona habitual de pesca del esturión no era natural, sino totalmente artificial. La construcción de azudes y presas propició que las aguas cercanas a estas construcciones fueran el lugar donde se desovaba, al no existir otra opción viable, porque no se podía remontar el río más allá, hacia el espacio natural de reproducción.

Las artes de pesca del esturión se diferencian de otras especies selectas y caras en pescaderías, como delfines. El alto valor comercial del sollo y, especialmente, el no ser considerado como un enemigo natural de pescadores, le daba un trato cuyo fin era que llegara la captura entera e inmaculada al mercado. La participación de varias familias de pescadores era necesaria para pescar con brugina o tirs plans, debido a su considerable longitud y al notable tamaño de las capturas. La falta de información al 
respecto en las fuentes analizadas impide, por ahora, precisar cuántas personas eran necesarias en esta actividad.

Por otra parte, aunque el esturión podía adquirirse desde finales de enero en los ríos, los cocineros profesionales, sabedores de su estacionalidad, preferían los sollos más grandes para preparar recetas y platos para sus señores, capturados entre los meses de abril y mayo. El precio del pescado venía, como demuestra Antonio Riera Melis, por su tamaño y gusto. En consecuencia, cuánto más grande el ejemplar, más caro era en una pescadería. Los cocineros respondían así al gusto de las élites, que demandaban no sólo un pescado, sino una forma de ostentar su riqueza, estatus y poder por medio de la exhibición en un banquete de un esturión de gran tamaño.

\section{REFERENCIAS BIBLIOGRÁFICAS}

Abad García, I., PeribáÑez Otero, J. (2006). La pesca fluvial en el reino de Castilla durante la Edad Media. En Vivir del agua en las ciudades medievales (Coord. María Isabel del Val Valdivieso). Valladolid: Universidad de Valladolid, Secretariado de Publicaciones e Intercambio Editorial.

Alanyà Roig, J. (2005). La carta cibariorum o Libre de les Viandes de la Canònica de Tortosa (1350). Acta Historia et Archeologica Medievalia, (26), 429-484.

Álvarez Fernández, M. (2009). Abastecimiento y consumo de pescado en Oviedo a finales de la Edad Media. En: La pesca en la Edad Media (pp. 56-71). Madrid: Universidad de Murcia.

Amargier, P. A. Gens de mer en méditerrannée dans les années 1375-1390. En Navigation et gens de mer en Mediterranée de la prehistoire à nos jours: actes de la table ronde du Groupement d'interet scientifique, Sciences humaines sur l'aire méditerranéene, Collioure, Septembre 1979 (pp. 68-83). Paris: Éditions du Centre National de la Recherche Scientifique, 1980.

ApArici Romero, F. (2012). La pesca durant l'edat mitjana a través de les fonts literàries catalanes. En J. Méndez Cabrera, D. A. Reinaldos Miñarro (Coords.), Nuevos estudios multidisciplinares sobre historia y cultura medieval: fuentes, metodología y problemas. Nous estudis multidisciplinaris sobre història i cultura medieval: fonts, metodologia i problemàtiques, (p. 13-24). Murcia, Centro de Estudios Medievales de la Universidad de Murcia, Universidad de Murcia. Servicio de Publicaciones. ARIÉ, R. (1982). España musulmana (siglos VIII-XV). Barcelona: Labor.

Aston, M. (1988). Mediaeval Fish Fisheries and Fish-ponds in England Part 1. London: BAR Publishing. https://doi.org/10.30861/9780860545095

Alcover Cateura, P. J. (2019). Els mercats alimentaris a través de la documentació municipal (segles XIV-XV). Barcelona: Universitat de Barcelona.

Alcover Cateura, P. J. (2022). Aproximación a la pesca, venta y consumo de delfín en la Corona de Aragón (siglos XIV-XV). Imago Temporis Medium Aevum, 15, s. n. 
Azevedo Pereira, O. N. (2012). Em torno da pesca, na costa Norte de Portugal, nos séculos finais da Idade Média (1292 - 1493). Porto: Universidade do Porto.

Aznar Vallejo, E. (2004). Marinos y pescadores. Medievalismo: Boletín de la Sociedad Española de Estudios Medievales, (13-14), 229-240.

BAJET Royo, M. (1994). El mostassaf de Barcelona i les seves funcions en el segle XVI. Edició del "Llibre de les Ordinations". Barcelona: Fundació Noguera.

BARrett, J. H, Locker, A. M., Roberts, C. M. (2005). The origins of intensive marine fishing in medieval Europe: English Evidence. Proceedings of the Royal Society of Biological Sciences, 271, 2417-2421. https://doi.org/10.1098/rspb.2004.2885

BARRIO BARRIO, J. A. (2020). La biografía social de los conversos de judíos valencianos. Una propuesta metodológica de investigación a partir de las fuentes inquisitoriales. Anales de la Universidad de Alicante. Historia Medieval, (21), 25-50. https://doi.org/10.14198/medieval.2019-2020.21.01

Bartosiewicz, L., Biller, A. Z., CSIPPÁn, P., DarócZi-Szabó, L., DarócZiSzabó, M., Gál, E., Kováts, I., Lyublyanovics, K., Ágnes Nyerges, É. (2018). Animal Exploitation in Medieval Hungary. En: J. Laszlovszky, B. Nagy, P. Szabó, A. Vadas, The Economy of Medieval Hungary (pp. 113-165). https://doi.org/10.1163/9789004363908_007

Bartosiewicz, L., Bonsall, C. (2008). Complementary taphonomies: Médiéval sturgeons from Hungary. En P. Béarez, Archéologie du poisson, 30 ans d'archéoichtyologie au CNRS: hommage aux travaux de Jean Desse et Nathalie Desse-Berset; XXVIIIe Rencontres Internationales d'Archéologie et d'Histoire d'Antibes; XIVth Icaz Fish Remains Working Group meeting (pp. 35-45). Paris: Antibes.

BATlle, C., PARÉS, A. (1986-1987). El Castell de Sitges a la mort de Bernat de Fonollar (1326). En M. Riu (Ed.), Fortaleses, torres, guaites i castells de la Catalunya Medieval. Acta Medievalia. Annexos d'Arqueologia Medieval. Annex 3 (pp. 153177). Barcelona: Universitat de Barcelona.

Baucells Reig, J. (1990). El Garraf i la Pia Almoina de la Seu de Barcelona: catàleg del fons en pergamí de l'Arxiu Capitular de la Catedral de Barcelona. Barcelona: Departament de Cultura de la Generalitat de Catalunya.

BAYERRI BERTOMEU, E. Historia de Tortosa y su comarca. Tortosa: Imprenta moderna de Algueró y Baiges, 1935-1960, 8 vols.

Bello León, J. M. (2009). Pescadores andaluces y canarios a final de la Edad Media. En: La pesca en la Edad Media (pp. 35-54). Madrid: Universidad de Murcia.

BLAIR, J. (2007). Waterways and Canal-Building in Medieval England. Oxford: Oxford University Press.

BRAmon, D. (2000). De quan érem o no musulmans: textos del 713 al 1010: continuació de l'obra de J.M. Millàs i Vallicrosa. Barcelona/Vic: IEC, Eumo.

BRAMON, D. (2017). Moros i catalans. La història menys coneguda dels sarraïns a Catalunya. Barcelona: Angle Editorial. 
Canellas, A. (1972). Colección diplomática del Concejo de Zaragoza, vol. I (años 1119-1276. Zaragoza: Universidad de Zaragoza.

Carreras CANDi, F. (1993). La navegació del riu Ebre. Notes històriques. Barcelona: Generalitat de Catalunya. Departament de Política Territorial i Obres Públiques. Direcció General de Ports i Costes.

Caruana Tomás, C. (1954). Estudio histórico y jurídico de la Albufera de Valencia. Su régimen y sus aprovechamientos. Valencia: Sucesor de Vives Mora.

Cave, R. C., Coulson, H. H. (1965). A Source Book for Medieval Economic History. New York: Biblo and Tannen.

Colón Domènech, G. (2001). Sobre els peixos gerret i xucla. Estudis romànics, (23), 91-101.

Cuellas Campodarbe, R. (Ed.) (2016). El Llibre de Costums, Privilegis i Ordinacions de la ciutat de Balaguer. Lleida: Universitat de Lleida.

Curto Homedés, A. (1995). El consum de peix a la Tortosa baix-medieval. En: $1^{o}$ Col.loqui d'Història de l'Alimentació a la Corona d'Aragó (pp. 149-166). Lleida: Institut d'Estudis Ilerdencs.

DAVIS, S. J. M. Faunal remains from Alcáçova de Santarém, Portugal. Lisboa: Instituto Portugués de Arqueologia, 2006.

De Castro Martínez, T. (2004). El abastecimiento alimentario en el reino de Granada (1482-1510). Granada: Universidad de Granada.

De los llanos Martínez Carrillo, M. (1997). Los paisajes fluviales y sus hombres en la baja Edad Media en 1 discurrir del Segura. Murcia: Universidad de Murcia.

De Oliveira Marques, A. H. R. (1987). Portugal na crise dos séculos XIV e XV. Lisboa: Editorial Presença.

Delsalle, L.-R. (2013). La pêche en Seine du XIIIe au XVIIe siècle. Lexploration de fief de l'Eau de l'abbaye de Saint-Ouen de Rouen. Études Normandes, (62/1), 95-118. https://doi.org/10.3406/etnor.2013.2892

Di SCHINo, J. (2010). Parola di pesce. Lo storione e il pescato sulla tavola rinascimentale di Bartolomeo Scappi cuoco segreto di Papa Pio V. En Valdo d'Arienzo y Biagio Di Salvia (Coords.), Pesci, barche, pescatori nell'area mediterranea dal medioevo all'està contemporanea. Atti del Quarto Convegno Internazionale di Studi della Pesca, (pp. 538-571). Milano: Franco Angeli.

Domezain, A. (2009). Main Steps and Proposals for a Recovery Plan of Sturgeon in the Guadalquivir River (Spain). En R. Carmona, A. Domezain, M. GarcíaGallego, J. A. Hernando, F. Rodríguez, M. Ruiz-Tejón, (Eds.), Biology, conservation and Sustainable Development of Sturgeons (pp. 423-452). Berlin: Springer. https://doi.org/10.1007/978-1-4020-8437-9_26

DufourCQ, C. E. La vida cotidiana de los árabes en la Europa medieval. Madrid: Ediciones Temas de Hoy, 1995. 
Duran, E. (Ed.) (1981). Cristòfor Puig. Los col.loquis de la Insigne Ciutat de Tortosa. Barcelona: Universitat de Barcelona.

Escolano, G. J. (1980). Historia de Valencia. Tomo Primero. Libros IV y V. Valencia: Colección Biblioteca Valenciana.

Felisati, D. (1998). In principio era il Po: storia, cultura, ambiente. Padua: Marsilio Editori.

Ferreira PRIEGE, E. M. (1984). Fuentes para la exportación gallega en la segunda mitad del siglo XV: el peatge de mar de Valencia. Santiago de Compostela: Universidad de Santiago.

Ferreira Priege, E. M. (1988). Galicia en el comercio maritimo medieval. Santiago de Compostela: Universidad de Santiago.

Ferreira Priege, E. M. (2009). Pesca y economía regional en Galicia. En: La pesca en la Edad Media (pp. 11-34). Madrid: Universidad de Murcia.

FlandRin, J. L. (1992). Structure des menus français et anglais aux XIVe et XVe siècles. En C.J. Lambert (Dir.), Du manuscrit à la table. Essais sur la cuisine au Moyen Âge et répertoire des manuscrits médiévaux contentant des recettes culinaires (pp. 173-192). Paris-Montreal: Université de Montreal.

FlandRIN, J. L. (1993). Diététique et gastronomie, XIVe-XVIIIe siècles. En R. Jansen-Sieben, F. Dealemans (Eds.), Vodeing en geneeskunde/Alimentation et medicine. Acten van het colloquium/Actes du colloque, Brussel-Bruxelles 12.10 .1990 (pp. 177192). Bruxelles: Algemeen Rijksarchief.

FlANDRIN, J. L. (2007). Arraiging the meal. A History of Table Service in France. Berkeley and Los Angeles: University of California Press.

Freire Moliner, M. J. (1995). El Libro registro de los privilegios de la Albufera de Valencia. Zaragoza: Anubar.

Furió, A., Garcia Oliver, F. (2007). Llibre d'establiments i ordenacions de la ciutat de València. I.: (1296-1345). València: Universitat de València.

GARCIA EDO, V. (2016). El Fuero de Zaragoza en el siglo XII (aproximación a su estudio). Revista de Dret Històric Català, (15), 165-197.

García Marsilla, J. V. (1993). La jerarquía de la mesa: los sistemas alimentarios en la Valencia bajomedieval. Valencia: Diputació de València.

García Marsilla, J. V. (2010). La taula del senyor duc. Alimentació i etiqueta a la cort dels ducs reials de Gandia. Gandia: CEIC Alfons el Vell.

García Marsilla, J. V. (2018). Food in the accounts of a travelling lady: Maria de Luna, queen of Aragon, in 1403. Journal of Medieval History, (44/5), 1-26. https://doi.org/10.1080/03044181.2018.1523066

GARCía SÁNCHEZ, E. La alimentación en la Andalucía Islámica. Estudio histórico y bromatológico. Carne, pescado, huevos, leche y productos lácteos. Andalucía Islámica. Textos y Estudios, IV-V (1983-1986), 237-278. 
Giagnacovo, M. (2002). Mercanti a tavola: prezzi e consumi alimentari dell'azienda Datini di Pisa (1383-1390). Firenze: Opus Libri.

Giagnacovo, M. (2018). Consumi e abitudini alimentari di una famiglia mercantile del basso Medioevo. La tavola di Francesco Datini (1367-1374). Nuova rivista storica, 102/1, pp. 229-264.

Granado Lorencio, C. (2002). Ecología de peces. Sevilla: Universidad de Sevilla.

GrAVA, Y. Marchands, pêcheurs et gens de mer sur les bords de l'Etang de Berre à la fin du Moyen Âge. En Navigation et gens de mer en Mediterranée de la prehistoire à nos jours: actes de la table ronde du Groupement d'interet scientifique, Sciences humaines sur l'aire méditerranéene, Collioure, Septembre 1979 (pp. 48-58). Paris: Éditions du Centre National de la Recherche Scientifique, 1980.

GRESSER, P. (2008). Pêche et pisciculture dans les eaux princières en Franche-Comté aux XIVe et XVe siècles. Turnhout: Brepols. https://doi.org/10.1484/M.BURG-EB.5.105849

Gual Camarena, M. (2020). Vocabulario del comercio medieval. http://www.um.es/lexico-comercio-medieval\%20 [Consulta: 16/12/2020].

Guerrero Navarrete, Y. (2009). Consumo y comercialización de pescado en las ciudades castellanas de la Baja Edad Media. En: La pesca en la Edad Media (pp. 235-262). Madrid: Universidad de Murcia.

Guix SugranyéS, J. M. (1963). El "Llibre de la Cadena de Reus". Règim jurídic de la vila en l'Edat Mitjana. Volum segon. Transcripció i glossari. Reus: Asociación de Estudios Reusenses.

Haidvogl, G., Lajus, D., Pont, D., Schmid, M. Jungwirth, M., Lajus, J. (2014). Typology of historical sources and the reconstruction of long-term historical changes of riverine fish: a case study of the Austrian Danube and northern Russian rivers. Ecology of Freshwater Fish, (23), 498 - 515. https://doi.org/10.1111/eff.12103

HeRnández Íñigo, P. (1997). La pesca fluvial y el consumo de pescado en Córdoba (1450-1525). Anuario de Estudios Medievales, (27), 1045-1116. https://doi.org/10.3989/aem.1997.v27.i2.632

Hinojosa Montalvo, J. (1987). La economía agropecuaria alicantina en tiempos de Alfonso X: aproximación a su estudio. Anales de la Universidad de Alicante. Historia Medieval, (6), 159-173. https://doi.org/10.14198/medieval.1987.6.08

Hinojosa Montalvo, J. R. (1995a). Comercio, Pesca y Sal en el Cap de Cerver (Orihuela) en la Baja Edad Media. Investigaciones Geográficas, (14), 191-204. https://doi.org/10.14198/INGEO1995.14.03

Hinojosa Montalvo, J. (1995b). Las tierras alicantinas en la Edad Media. Alicante: Instituto de Cultura "Juan Gil-Albert," Diputación de Alicante.

Hinojosa Montalvo, J. R. (1996). Abastecimiento y consumo de pescado en tierras alicantinas durante la Baja Edad Media. En La Mediterrània, àrea de convergència de sistemes alimentaris (segles V-XVIII): XIV Jornades d'Estudis Històrics Locals, 
Palma, del 29 de novembre al 2 de desembre de 1995 (pp. 209-222). Palma, Institut d'Estudis Baleàrics.

Hinojosa Montalvo, J. R. (1995c). Comer y beber en Alicante en la Edad Media. En: $1^{\circ}$ Col.loqui d'Història de l'Alimentació a la Corona d'Aragó (pp. 507-525). Lleida: Institut d'Estudis Ilerdencs.

Hinojosa Montalvo, J., Barrio BarRio, J. A. (1992). Las sisas en la Gobernación de Orihuela durante la Baja Edad Media, Anuario de Estudios Medievales, (22), 535- 579. 536-543. https://doi.org/10.3989/aem.1992.v22.1081

HofFmann, R. C. (2016). Fishers' Craft and Lettered Art: Tracts on Fishing from the End of the Middle Ages. Toronto: University of Toronto Press.

ICART, J. (Ed.) (1982). Ordinacions i crides de la ciutat de Tarragona (segles XIV-XVII). Tarragona: Ajuntament de Tarragona.

IGUAL Luis, D. (2007). El tráfico de pescado en la España meridional y su proyección hacia Italia durante el siglo XV. En G. Doneddu, (Coord.), Terzo convegno nazionale di Storia della Pesca. La pesca nel Lazio. Storia, economia, problemi attuali (pp. 277-309). Roma: Ed. Scientifica.

IGUAL Luis, D. (2010). Pesca y pescadores en el reino de Valencia (siglos XIII-XV). En V. d'Arienzo, B. Di Salvia (Coords.), Pesci, barche, pescatori nell'area mediterranea dal medioevo all'està contemporanea. Atti del Quarto Convegno Internazionale di Studi della Pesca (pp. 68-84). Milano: Franco Angeli, 2010.

IGUAL LuIS, D. (2020). Proyección marítima y sectores laborales en una ciudad del siglo XV: Valencia, de la pesca a la construcción naval. En M. D. González Guardiola, D. Igual Luis, (Eds.), El mar vivido. Perfiles sociales de las gentes de mar en la larga duración (siglos XV-XXI) (pp. 49-70). Cuenca: Ediciones de la Universidad de Castilla-La Mancha. https://doi.org/10.18239/estudios_2020.170.03

JOHNSON, T. (2019). Law in common: Legal Cultures in Late-Medieval England. Oxford: Oxford University Press. https://doi.org/10.1093/oso/9780198785613.001.0001

Jones, E. T., LaUghton, J., Clark, P. (2000). Northampton in the Late Medieval Ages: The Archeology and History of a Midland Town. Leicester: University of Leicester. KowAleski, M. (2000). The Expansion of the South-Western Fisheries in Late Medieval England. The Economic History Review, (53/3), pp. 429-454. https://doi.org/10.1111/1468-0289.00166

KOWALESKI, M. (2001). The haverner's Accounts of the Earldom and Duchy of Cornwall, 1287-1356. Devon: Devon and Cornwall Record Society.

KOWALESKI, M. (2010). The Seasonality of fishing in medieval Britain. En S. G. Bruce (Ed.), Ecologies and economies in medieval and early modern Europe: studies in environmental history for Richard C. Hoffmann (pp. 23-28). Leiden: Brill.

KowAlESKI, M. (2015). Coastal Communities in Medieval Cornwall. En P. Payton, A. Kennerley, H. Doe (Coord.), The maritime history of Cornwall (pp. 43-59). Exeter: University of Exeter Press. 
LANCONELli, A. (1985). Gli statuta pescivendulorum urbis (1405): note su comercio del pesce a Roma fra XIV e XV secolo. Archivio della Società Romana di Storia Patria, 108, pp. 83-131).

Lanconelli, A. (2005). Il commercio del pesce a Roma nel tardo Medioevo. En A. Esposito, L. Palermo, [Coords]. Economia e società a Roma tra Medioevo e Rinascimento: studi dedicati ad Arnold Esch (pp. 181-204). Roma: Viella.

LÉVI-Provençal, E. (1957). España musulmana: hasta la caída del Califato de Córdoba, 711-1031 de J.C. Madrid: Espasa Calpe.

Lleonart, J., Maynou, F., Salicrú i Lluch, R. (2018). Marine species and their selling prices in the Crown of Aragon. An initial approach with some examples from the 14th to the 17 th centuries". En G. Buti, D. Faget, O. Raveux, R. Solène, (Dirs.), Moissonner la mer. Économies, sociétés et pratiques halieutiques méditerranéennes (XVe-XXIe siècle) (pp. 159-173). Paris: Karthala.

Lloris, D., Rucabado, J., Cerro, lluís del., Demestre, M., Portas, F., Roig, A. (1984). Tots els peixos del mar català I. Llistat de cites i referències. Barcelona: Societat Catalana d'Ictiologia i Herpetologia.

LOCKER, A. M. (2000). The role of stored fish in England 900-1750AD; the evidence from historical and archaeological data. Southampton: University of Southampton, Deparment of Archeology.

Ludwig, A., Morales-Muñiz, A., Rosselló-IzQuierdo, E. (2011). Sturgeon in Iberia from Past to Present. En P. Williot, E. Rochard, N. Desse-Berset, F. Kirschbaum, J. Gessner (Eds.), Biology and conservation of European Sturgeon Acipenser sturia L. 1758. The Reunion of the European and Atlantic Sturgeon (pp. 147-152). Berlin: Springer.

Macheridis, S. HAnsson, M. C., Foley, B. P. (2020). Fish in a barrel: Atlantic sturgeon (Acipenser oxyrinchus) from the Baltic Sea wreck of the royal Danish flagship Gribshunden (1495). Journal of Archaeological Science: Reports, (33), 10241080. https://doi.org/10.1016/j.jasrep.2020.102474

Malpica Cuello, A. (1984). El pescado en el reino de Granada a fines de la Edad Media: especies y nivel de vida. En Denis Menjot (Ed.), Actes du colloque du Centre d'Etudes Médiévales de Nice (1982). Centre d'Etudes Médiévales de Nice 1: Aliments et société. 2. Régimes alimentaires (Vol. 1, pp. 103-117). Paris: Les Belles Lettres.

Malpica Cuello, A. (2009). La pesca en el mar de Alborán en época nazarí. En: La pesca en la Edad Media (pp. 193-220). Madrid: Universidad de Murcia.

MARCO BAIDAL, J. (1960). El Turia y el hombre ribereño. Valencia: Instituto de Estudios Ibéricos y Etnología Valenciana, Institución Alfonso el Magnánimo.

MEDRANO FERnÁNDEZ, V. La pesca y el comercio de pescado en la frontera castellanoportuguesa al final de la Edad Media. En: La pesca en la Edad Media (pp. 221234). Madrid: Universidad de Murcia. 
Mercader, Ll., LloRis, D., Rubacabo, J. (2003). Tots els peixos del mar català. Diagnosis i claus d'identificació. Barcelona: Institut d'Estudis Catalans (IEC).

Mollat DU Jourdin, M. (Ed.) (1987). Histoire des pêches maritimes en France. Paris: Privat.

Momblanch y GonzÁlBez, F. (2003). Historia de la Albufera de Valencia. Valencia: Ayuntamiento de Valencia.

MONTANARI, M. (1985). Gli animale e l'alimentazione umana. En L'Uomo di fronte al mondo animale nell'alto Medioevo: 7-13 aprile 1983 (p. 619-672). Spoleto: Centro italiano di studi sull'alto Medioevo.

Montanari, M. (1988). Alimentazione e cultura nel Medioevo. Firenze: Laterza.

MONTANARI, M. (1989). Nuovo convivio: storia e cultura dei piaceri della tavola dall'antichità al medioevo. Firenze: Laterza.

Montanari, M. (1993). La fame e l'abbondanza. Storia dell'alimentazione in Europa. Firenze: Laterza.

Montanari, M. (2019). Gusti del medioevo. I prodotti, la cucina, la tavola. Firenze: Laterza.

Morales Muñiz, D. C., Roselló Izquierdo E., Morales MuñIz, A. (2009). Pesquerías medievales hispanas: las evidencias arqueofaunísticas. En: La pesca en la Edad Media (pp. 145-165). Madrid: Universidad de Murcia.

Morales-Muñiz, A. Rosselló-Izquierdo, E., Lentacker, A., Morales-Muñiz, D. C. (1994). Archeological research in Medieval Iberia: Fishing and Fish trade on Almohad Sites, Trabalhos de Antropologia e Etnologia, (34/1-2), 453-475.

MuntanÉ I SANTIVERI, X. Llibres de bans de Tàrrega, segle XIV. Urgell: Arxiu Comarcal de l'Urgell.

Mutgé Vives, J. (2004). Política, urbanismo y vida ciudadana en la Barcelona del siglo XIV. Barcelona: IMF-CSIC.

NAVARro, M. A. (Ed.) 1990. Risāla fì awqāt al-sana. Madrid: CSIC.

NiENHUIS, P.H. (2008). Environmental History of the Rhine-Meuse Delta: An ecological story on evolving human-environmental relations coping with climate change and sea-level rise. London: Springer. https://doi.org/10.1007/978-1-4020-8213-9

Ordeix, M., Solà, C., Bardina, M., Casamitjana, A., Munné, A. (Eds.) (2014). Els peixos dels rius i les zones humides de Catalunya. Qualitat biologica i connectivitat fluvial. Vic: Agència Catalana de l'Aigua, Museu del Ter, Eumo Editorial.

PucCi Donati, D. (2017). Il mercato del pesce. Regolamentazione dei prezzi a Bologna nel Medioevo (secc. XIII-XV). En: I prezzi delle nell'èta preindustriale/The prices of things in Pre-industrial times. Serie II-Atti delle "Settimane di Studi" e altri Convegni. Firenze: Firenze University Press, Fondazione Instituto Internazionale di Storia Economica "F. Datini" Prato.

Querol Gómez, A. (2004). Processos i canvis al territorio del delta de l'Ebre. Tarragona: Cossetània Edicions. 
Querrien, A. (2003). Pêche et consommation du poisson en Berry au Moyen Âge. Bibliothèque de l'École des chartes, (161-2), 409-435.

https://doi.org/10.3406/bec.2003.463623

RiERA MELIS, A. (1994). Estructura social y sistemas alimentarios en la Cataluña bajomedieval. Acta Historia et Archeologica Medievalia, (14), 193-217.

RiERA MELIS, A. (1996). Jerarquía social y desigualdad alimentaria en el Mediterráneo Nor-occidental en la Baja Edad Media. La cocina y la mesa de los estamentos privilegiados. Acta Historia et Archeologica Medievalia, (16), 181-205.

Riera Melis, A. (2009). La pesca en el Mediterráneo Noroccidental durante la Baja Edad Media. En: La pesca en la Edad Media (pp. 121-143). Madrid: Universidad de Murcia.

Riera Melis, A. (2013). Gastronomía y política en los banquetes cortesanos de la Baja Edad Media. En M. García Guatas, E. Piedrafita, J. Barbacil (Coords.), La alimentación en la Corona de Aragón (siglos XIV-XV). Actas del Simposio organizado por la Academia Aragonesa de Gastronomía en el sexto centenario del Compromiso de Caspe, que tuvo lugar en el antiguo salón de Plenos de la Diputación de Zaragoza, durante los días 23 y 24 de marzo de 2012 (pp. 65-98). Zaragoza: Institución "Fernando el Católico" CSIC, Excma. Diputación de Zaragoza.

Riera Melis, A. (2014). El context històric del receptaris medievals catalans 1. L'evolució de les estructures productives i de distribució d'aliments. En J. Santanach, M. Barrieras., A. Riera Melis, F. Solé i Parellada, C. Duarte, R. A. Banegas, (Eds.), Llibre de Sent Soví (pp. 23-80). Barcelona: Barcino.

RiERA MElis, A. (2016). El context històric del receptaris medievals catalans. 2. La cuina i la taula de la noblesa: l'ostentació de la qualitat. En J. Santanach, M. Barrieras., A. Riera Melis, Fundació Alícia, (Eds.), Llibre d'aparellar de menjar (pp. 21-52). Barcelona: Barcino.

Riera Melis, A. (2017). El context històric del receptaris medievals catalans. 3. Lalimentació dels canonges regulars dels capítols catedralicis: uns comensals cultes i exigents. En Santanach, J., Barrieras. M., Riera Melis, A., Fundació Alícia (Eds.), Llibre de totes maneres de potatges (pp. 21-66). Barcelona: Barcino.

RiERA MELIS, A. (2018). El context històric del receptaris medievals catalans. 4. La taula del príncep: gastronomía, representació i política. En Santanach, J., Barrieras. M., Riera Melis, A., Fundació Alícia, (Eds.), Mestre Robert. Llibre del coc (pp. 17-84). Barcelona: Barcino.

Roca Traver, F. A. (1907). El Mustaçaf de Castellón y el Libre de Mustaçafia. Boletín de la Sociedad Castellonense de Cultura, s. n.

Roca Traver, F. A. (1952). El Mustaçaf de Castellón y el Libre de Mustaçafia. Boletín de la Sociedad Castellonense de Cultura, 455-492.

Roca Traver, F. A. (1973). El Mustaçaf de Castellón y el Libre de la Mustaçaffia. Valencia: Diputación de Valencia. 
Rodrigo Estevan, M. L. (2009). Fresco, frescal, salado, seco, remojado. Abasto y mercado de pescado en Aragón (siglos XII-XV). En B. Arízaga Bolumburu, J. A. Solórzano Telechea, Alimentar la ciudad en la Edad Media: Nájera, Encuentros Internacionales del Medievo 2008, del 22 al 25 de julio de 2008 (pp 547-577). Logroño: Instituto de Estudios Riojanos.

Rosselló Verger, V. M. (1995). L'Albufera de València. Barcelona: Publicacions de l'Abadia de Montserrat.

Rosselló-IzQuierdo, E., Morales-Muñiz, A., Morales-Muñiz, D. C. (1994). La Cartuja/Spain: Anthropogenic Ichthyocenosis of culinary nature in a paleocultural context. Offa, (51), 323-331.

SÁNCHEZ QuiÑonES, J. (2006). Pesca y trabajo en el Reino de Toledo. La cuenca alta y media del Tajo en los siglos XII a XVI. Anuario de Estudios Medievales, (26/1), 145-169. https://doi.org/10.3989/aem.2006.v36.i1.8

SANCHIS IBOR, C. (2011). Regadiu i canvi ambiental a l'Albufera de València. València: Universitat de València.

SANMARTín ARCE, R. (1982). La Albufera y sus hombres. Madrid: Akal.

SANTAMARÍA, A. (1966). Aportación al estudio de la economía de Valencia durante el siglo XV. Valencia: Instituto Valenciano de Estudios Históricos, Institución Alfonso El Magnánimo, Diputación Provincial de Valencia.

SANTANACH, J. (2017). «Suplic les vostres benignitats que prengau mon servici en grat»: sobre la composició i el contingut del Llibre de potatges, En Mònica Barrieras, Antonio Riera Melis, Joan Santanach, (Eds.), Llibre de totes maneres de potatges (pp. 67-91). Barcelona: Barcino, 2017.

SALICRÚ LlUCH, R. (1995). El tràfic de mercaderies a Barcelona segons els comptes de la lleuda de Mediona (febrer de 1434). Barcelona: CSIC.

SERRANO LARRÁYOZ, F. (2005). La mesa del rey: cocina y régimen alimentario en la corte de Carlos III el Noble de Navarra (1411-1425). Pamplona: Gobierno de Navarra, Departamento de Educación y Cultura.

SeSma MuÑoz, J. (2005). Huesca, ciudad mercado de ámbito internacional en la baja edad media según los registros de su aduana. Zaragoza: Universidad de Zaragoza.

Sesma Muñoz, J. (2006). La vía del Somport en el comercio medieval de Aragón : los registros de las aduanas de Jaca y Canfranc de mediados del siglo XV. Zaragoza: Universidad de Zaragoza.

SeSMA MUÑOZ, J. (2010). El tráfico mercantil por las aduanas de Ribagorza (14441450): producción y comercio rural en Aragón a finales de la Edad Media. Zaragoza: Universidad de Zaragoza.

SOBReQuÉS CAllicó, Jaume (2008). Estudis d'història de Catalunya: Edat mitjana, edat moderna, El pactisme. Barcelona: Editorial Base.

StoufF, L. (1970). Ravitaillement et alimentation en Provence aux XIVe et XVe siècles. Paris-La Haye: CNRS, École Practique des Hautes Études. https://doi.org/10.1515/9783111677446 
Stunault, C. (2019). Les repas des capitouls à l'appui des comptes de la municipalité de Toulouse (1444-1470). Comptabilité (s), revue d'histoire des comptabilités, (19). http://journals.openedition.org/comptabilites/3882.

SweEtinburgh, S. (2020). Fishermen and their families in late medieval and Tudor Kent. En C. Jowitt, C. Lambert, S. Mentz, (Eds.), The Routledge Research Companion to Marine and Maritime Worlds, 1400-1800: Oceans in Global History and Culture Routledge (pp. 80-99). London: Routledge. https://doi.org/10.4324/9781003048503-9

TAVARES, A. Pesca e comércio de peixe em portugal da Idade Média até aos primórdios do século XVI: conflictos e soluçoes. En F. J. Díaz Marcilla, J. T. García, Y. Sobral dos Santos (Eds.), Global History, Visual Culture and Itinerancies: Changes and Continuities (pp. 44-57). London: Cambrige Scholars Publishing.

TeÑa GarCía, M. S. (1995). Composición social y articulación interna de las cofradías de pescadores y mareantes (Un análisis de la explotación de los recursos marítimos de Castilla durante la Baja Edad Media). Espacio, tiempo y forma. Serie III, Historia Medieval, (8), 111-134. https://doi.org/10.5944/etfiii.8.1995.3597

Thomazi, A. (1947). Histoire de la pêche des âges de la pierre à nos jours. Paris: Payot. Toledo Jordán, J. M. (1998). El Cádiz Andalusí: (711-1485). Cádiz: Diputación de Cádiz, Servicio de Publicaciones.

Trenchs Odena, J. (1995). El peix a la taula de la princesa Mata d'Armanyac: els capritxos i gustos d'una infanta. En: $1^{\circ}$ Col.loqui d'Història de l'Alimentació a la Corona d'Aragó (pp. 309-328). Lleida: Institut d'Estudis Ilerdencs.

VALlbÉ, J. (2003). Abderramán III: califa de España y Occidente (912-961). Barcelona: Ariel.

Velasco Marcos, J. C. Los peces de la provincia de Salamanca. Atlas de distribución. Salamanca: Universidad de Salamanca.

Venditelli, M. (1992). Diritti e impianti di pesca degli enti ecclesiastici romani tra X e XIII secolo. Mélanges de l'École française de Rome. Moyen-Age, (102/2), 287430. https://doi.org/10.3406/mefr.1992.3251

VICTOR, S. (2018). Réglementer pour protéger? Le livre du Mostassaf géronais comme outil de protection du consommateur sur le marché (XVe siècle). En Pétrowiste, J., Lafuente Gómez, M. (Dir.), Faire son marché au Moyen Âge, Méditerranée Occidentale, XIIIe-XVIe siècle, Actes du colloque de mars 2015 (pp. 227-238). Madrid: Casa de Velazquez.

Williot, P., Rouault, T., Brun, R., Gressner, J. (2011). Characteristics of the Reproductive Cycle of Wild Acipenser sturio. En P. Williot, E. Rochard, N. Desse-Berset, F. Kirschbaum, J. Gessner (Eds.), Biology and conservation of European Sturgeon Acipenser sturia L. 1758. The Reunion of the European and Atlantic Sturgeon (pp. 165-174). Berlin: Springer. https://doi.org/10.1007/978-3-642-20611-5_12 